\title{
Renal Vein Thrombosis and Pulmonary Embolism Secondary to Vaccine-induced Thrombotic Thrombocytopenia (VITT)
}

\author{
Nikita Cliff-Patel, Lindsay Moncrieff, Veqas Ziauddin \\ East Surrey Hospital, Redhill, Surrey, United Kingdom
}

Received: 04/06/2021

Accepted: 09/06/2021

Published: 30/06/2021

How to cite this article: Cliff-Patel N, Moncrieff L, Ziauddin V. Renal vein thrombosis and pulmonary embolism secondary to vaccine-induced thrombotic thrombocytopenia. EJCRIM 2021;8: doi:10.12890/2021_002692.

Conflicts of Interests: The authors declare there are no competing interests.

This article is licensed under a Commons Attribution Non-Commercial 4.0 License

\section{ABSTRACT}

The Medicines and Healthcare products Regulatory Agency (MHRA) of the UK has approved the use of three vaccines to combat COVID-19 (SARS-CoV-2). There have been rare reports of thrombosis after vaccination with the AstraZeneca vaccine. We present three cases of vaccine-induced thrombotic thrombocytopenia (VITT) in one UK district general hospital following administration of this vaccine. Two of the patients had asymptomatic pulmonary emboli, while the other is the first known case of both renal vein thrombosis and pulmonary embolism.

\section{LEARNING POINTS}

- Vaccine-induced thrombotic thrombocytopenia (VITT) can be associated with unusual and multiple sites of thrombosis.

- Clinicians should have a low threshold for requesting anti-PF4 antibody tests and imaging (especially pulmonary imaging) in thrombocytopenic patients after administration of the AstraZeneca vaccine.

- We describe a localised cluster of VITT despite its rarity according to current statistics, highlighting the need for an efficient data collection system to ensure the incidence of VITT is accurately reported.

\section{KEYWORDS}

Vaccine-induced thrombotic thrombocytopenia, renal vein thrombosis, pulmonary embolism

\section{CASE DESCRIPTION}

A 33-year-old man presented with a 1-week history of back pain, haematuria, headache and right lower leg pain. He had received the AstraZeneca vaccine 4 weeks earlier. Examination revealed bilateral flank and right lumbar tenderness. There were no clinical signs of deep vein thrombosis (DVT). Blood analysis showed a mildly elevated white cell count and acute kidney injury (AKI). Examination was otherwise unremarkable apart from a low-grade fever. Oxygen saturation levels were $96 \%$ on air. A non-contrast CT scan of the urinary tract showed inflammatory changes in the right kidney likely related to a recently passed kidney stone and pyelonephritis. The patient was treated for pyelonephritis and admitted to hospital.

In view of his recent vaccine and low platelet levels, tests for anti-platelet factor 4 antibodies (PF4) and D-dimer were requested. D-dimer was elevated at $>20 \mathrm{mg} / \mathrm{l}$ (normal $<0.50 \mathrm{mg} / \mathrm{l}$ ) and anti-PF4 antibodies were positive at 1.35 (normal range $<0.4$ ).

Ultrasound of the right lower leg was negative for DVT and an intracranial CT venogram was negative for venous sinus thrombosis (VST). Even though there were no obvious symptoms of a pulmonary embolism (PE), a CT pulmonary angiogram (CTPA) was requested and demonstrated a PE at the bifurcation of the left pulmonary artery. 
A repeat $\mathrm{CT}$ scan of the urinary tract with contrast demonstrated a right renal vein thrombus extending into the inferior vena cava with some renal infarction (Fig. 1). The patient occasionally had temperature spikes. However, multiple blood and urine cultures were negative, so it was considered that the temperature spikes were likely an inflammatory response secondary to renal vein thrombosis. The patient's kidney function and inflammatory markers improved prior to discharge.

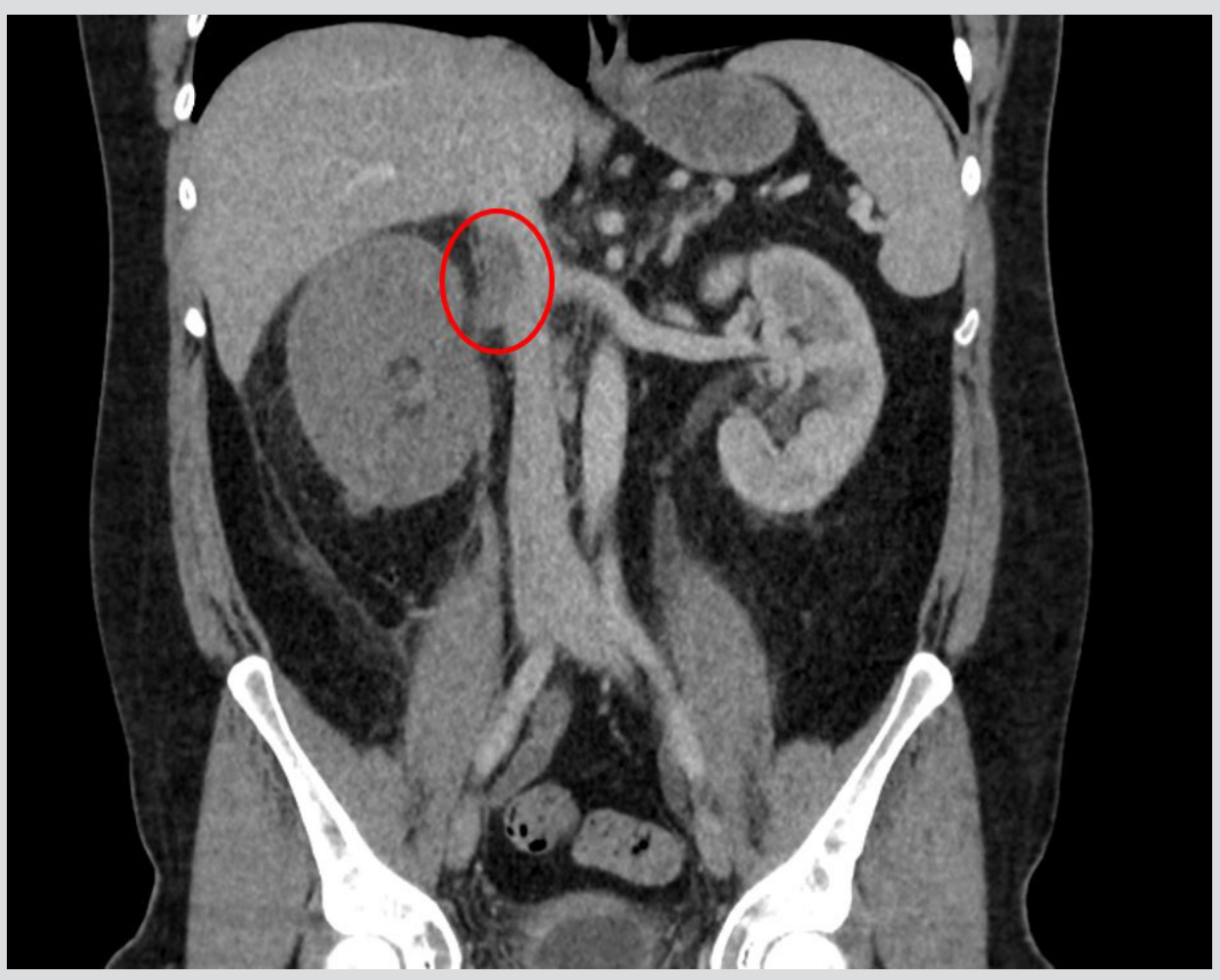

Figure 1. Thrombosis of the right renal vein extending into the inferior vena cava and infarct of the right kidney

In the same month, two additional patients were diagnosed with VITT. Both presented following vaccination with the AstraZeneca vaccine and were found to be thrombocytopenic. One was a 28-year-old man who presented with sudden-onset back pain and lower limb weakness. He presented 8 days after vaccination and was found to have bilateral PEs and left proximal DVT, despite having normal oxygen saturations and not showing any typical PE symptoms.

Our last patient's presentation was more typical. He was a 61-year-old man with a history of exertional dyspnoea and pleuritic chest pain 10 days after receiving the AstraZeneca vaccine. CTPA revealed extensive bilateral PEs. Both patients had elevated D-dimers and positive anti-PF4 antibodies. Neither patient had previously been exposed to heparin.

All three patients were treated similarly: they were commenced on intravenous (IV) argatroban and intravenous immunoglobulin (IVIg). IVIg was stopped when the platelet counts normalised (typically in 2-5 days). Warfarin was then started. Argatroban infusion was stopped once the patient was established on warfarin. All were discharged to haematology outpatient follow-up.

\section{DISCUSSION}

Between 9 December 2020 and 5 May 2021, an estimated 30.8 million AstraZeneca vaccines were administered in the UK [1]. The MHRA received over 260 reports of VITT following the first dose of the AstraZeneca vaccine during this period. The Joint Committee on Vaccination and Immunisation (JCVI) advises that adults under 40 years of age should be offered an alternative vaccine.

The overall risk of VITT following administration of the AstraZeneca vaccine is low (10.9 cases per million doses). Despite this, we have treated three cases of VITT in a single UK district general hospital within a period of 7 weeks. The patients presented to hospital 1-4 weeks after their first dose of the AstraZeneca vaccine. Positive anti-PF4 antibodies were detected in all three cases.

There are similarities between VITT and heparin-induced thrombocytopenia (HIT), which also presents with raised anti-PF4 antibodies ${ }^{[2]}$. 
The literature suggests activation of anti-PF4 antibodies due to the vaccine, triggering platelet aggregation and subsequently clot formation ${ }^{[2]}$. A similar mechanism has been reported in isolated cases of 'spontaneous' HIT syndrome (i.e., absence of heparin exposure), most commonly after orthopaedic surgery ${ }^{[2]}$.

VITT has been reported in rare locations such as cerebral venous sinuses, and splenic, hepatic and portal veins ${ }^{[3]}$. To the best of our knowledge, we present the first published case of a patient with both renal vein thrombosis and pulmonary embolism following vaccination with the AstraZeneca vaccine. It is important to note the atypical presentations of PEs in two of the presented cases of VITT with no respiratory symptoms.

All three patients received treatment as recommended by the UK's Expert Haematology Panel ${ }^{[3]}$. Given the similarities between HIT and VITT, patients should be treated with a non-heparin anticoagulant. Immunoglobulins inhibit the activation of platelets by anti-PF4 antibodies and are very effective at normalising platelet counts ${ }^{[2]}$. When bridging patients with warfarin, clinicians should aim for a higher target INR due to the recognised influence of IV argatroban on INR readings ${ }^{[4]}$.

It is important to acknowledge the raised risk of thrombosis due to COVID-19 infection itself. A meta-analysis of 42 studies published in 2020 evaluated 8271 COVID-positive patients and showed an overall thromboembolism rate of $21 \%$, which increased to $31 \%$ in the Intensive Care Unit setting ${ }^{[5]}$.

In conclusion, these cases should prompt clinicians to maintain a low threshold for requesting anti-PF4 antibody tests and imaging (especially pulmonary imaging) in thrombocytopenic patients presenting with any symptoms after AstraZeneca vaccination.

\section{REFERENCES}

1. Vagnoni C. Covid-19 vaccines safety and blood clots. 19 May 2021. Available from: https://post.parliament.uk/covid-19-vaccines-safety-and-blood-clots/ (accessed 25 May 2021).

2. Greinacher A, Thiele T, Warkentin TE, Weisser K, Kyrle PA, Eichinger S. Thrombotic thrombocytopenia after ChAdOx1 nCoV-19 vaccination. NEngl J Med 2021;384(22):20922101.

3. British Society for Haematology. Guidance produced from the Expert Haematology Panel (EHP) focused on Covid-19 vaccine induced thrombosis and thrombocytopenia (VITT). 7 April 2021. Available from: https://b-s-h.org.uk/media/19530/guidance-version-13-on-mngmt-of-thrombosis-with-thrombocytopenia-occurring-after-c-19vaccine_20210407.pdf (accessed 25 May 2021).

4. Hursting MJ, Lewis BE, Macfarlane DE. Transitioning from argatroban to warfarin therapy in patients with heparin-induced thrombocytopenia. Clin Appl Thromb Hemost 2005;11:279-287.

5. Malas MB, Naazie IN, Elsayed N, Mathlouthi A, Marmor R, Clary B. Thromboembolism risk of COVID-19 is high and associated with a higher risk of mortality: a systematic review and meta-analysis. EClinicalMedicine 2020;29:100639. 